\title{
Recent Statistics on the Growth of the KI Journal
}

\author{
Ubbo Visser ${ }^{1}$
}

Published online: 7 May 2015

(C) Springer-Verlag Berlin Heidelberg 2015

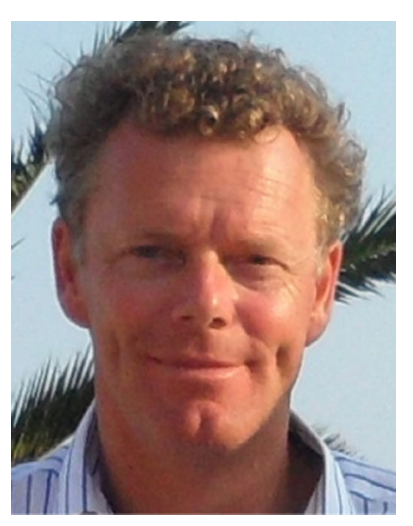

\section{Dear readers,}

We started to publish our articles at the SpringerVerlag with issue 01/2010. Five years have passed and it is time to reflect that decision. One way of reflection is to evaluate the publisher from the author's and editor's point of view. Another perspective is the inverse: what does the publisher think about their decision to take on the KI? And it is the latter that I would like to talk about today.

Springer presented interesting statistics during our last editor's meeting in Kaiserslautern in March 2015. The following remarks are based on that presentation. It takes the years 2011-2014 into account.

The production volume between 2012 and 2014 reveal that 60 articles per year were accepted for publication on average. This holds for both the printed version and the Online First version. A closer look on the 2014 data shows that each volume contains 85 pages on average. This is similar to other years at Springer and a little higher compared to the years with the BöttcherIT Verlag before 2010.

An interesting and significant information came out of the discussion about the production turnaround time. This is the average time between Springer receiving an accepted article and the Online First Publication. The years

Ubbo Visser

visser@cs.miami.edu

1 Department of Computer Science, University of Miami, Coral Gables, FL 33146, USA
2012-2014 show a production turnaround time between 13 and 22 days. This means that the entire publication time between the submission of an article and the Online First at Springer is comparable or less than most of the major conference publications. This is an excellent argument to publish more papers with our journal!

Online deals get more and more popular compared to the typical institutional subscriptions when it comes to article circulation. I suppose that this trend is not unique to our journal and publisher.

The statistics also show that the full-text download rate nearly doubled from 2011 to 2014. This is an indication for the positive impact of an international publisher like Springer. Consequently, the regional distribution of the fulltext downloads have also expanded: data from 2012 to 2014 show that more than half of the downloads were initiated from readers in Europe; 56 \% in 2012 with $31 \%$ from areas in Asia-Pacific, and $9 \%$ from North-America. Years 2013 and 2014 show an increasing full-text download trend from North-American readers ( $14,13 \%$, respectively), which can be interpreted as a growing impact of our journal in that area.

Overall, Springer is more than happy with both the quality and the distribution of this journal, especially because of the positive trend during the past few years. Good news for all of us!

And now it is time to wish you a pleasant reading with the new issue of Künstliche Intelligenz-Health and Wellbeing!

Kind regards,

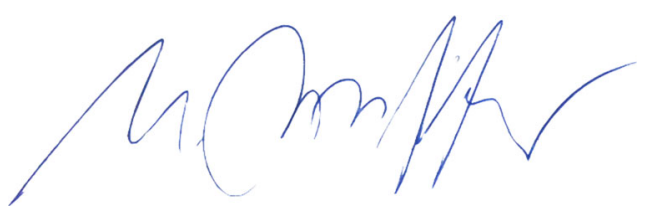




\section{Forthcoming Special Issues}

\subsection{Higher-Level Cognition and Computation}

Human higher-level cognition is a multi-faceted and complex area of thinking which includes the mental processes of reasoning, decision making, creativity, and learning among others. Logic, understood as a normative theory of thinking, has a widespread and pervasive effect on the foundations of cognitive science. However, human reasoning cannot be completely described by logical systems. Sources of explanations are incomplete knowledge, incorrect beliefs, or inconsistencies. Still, humans have an impressive ability to derive satisfying, acceptable conclusions. Generally, people employ both inductive and deductive reasoning to arrive at beliefs; but the same argument that is inductively strong or powerful may be deductively invalid. Therefore, a wide range of reasoning mechanism has to be considered, such as analogical or defeasible reasoning.

The topics of interest include, but are not limited to:

- Analogical reasoning

- Common sense and defeasible reasoning

- Deductive calculi for higher-level cognition

- Inductive reasoning and cognition

- Preferred mental models and their formalization

- Probabilistic approaches of reasoning.

The Künstliche Intelligenz journal, which is published and indexed by Springer, supports the following lists of formats: Technical contributions, research projects, discussions, dissertation abstracts, conference reports and book reviews. If you are interested in contributing to this special issue, please contact one of the guest editors:

\section{Dr. Marco Ragni}

University of Freiburg

Center for Cognitive Science

Institute of Computer Science and Social Research

Friedrichstr. 50

D-79098 Freiburg, Germany

ragni@cognition.uni-freiburg.de

portal.uni-freiburg.de/cognition/members/ragni

\section{Prof. Frieder Stolzenburg}

Harz University of Applied Sciences

Automation \& Computer Sciences Dep.

Friedrichstr. 57-59

38855 Wernigerode, Germany

fstolzenburg@hs-harz.de

fstolzenburg.hs-harz.de

\subsection{Advances in Autonomous Learning}

Autonomous learning research aims at understanding how adaptive systems can efficiently learn from the interaction with the environment, especially by having an integrated approach to decision making and learning, allowing systems to decide by themselves on actions, representations, hyper-parameters and model structures for the purpose of efficient learning. It addresses challenges such as how to autonomously learn representations for efficient model use, how to arrive at suitable cost functions from meta-objectives (generalizing inverse RL), how to autonomously choose model structures and hyper-parameters in possibly non-stationary environments, or how to design efficient actor-reward strategies which generalize across tasks. Application scenarios which require these type of complex models span high-impact domains such as robotics, lifelong learning, intelligent tutoring, or big data analytics.

We invite contributions related to the following nonexhaustive list of topics:

- Autonomous learning of rich data representations,

- Active learning in structured (e.g. hybrid, relational) interactive domains,

- Learning models with autonomous complexity adaptation,

- Transfer learning,

- Structure learning,

- Statistical relational learning,

- Theoretical advances to measure model autonomy,

- Applications and project reports in the field of autonomous learning.

The Künstliche Intelligenz journal, which is published and indexed by Springer, supports the following lists of formats: technical contributions, research projects, discussions, dissertation abstracts, conference reports and book reviews. If you are interested in contributing to this special issue, please contact one of the guest editors:

Prof. Barbara Hammer

Universität Bielefeld

D-33594 Bielefeld

Prof. Marc Toussaint

Universität Stuttgart

D-70569 Stuttgart

\subsection{Companion Technologies}

At present, we observe a rapid growth in the development of increasingly complex "intelligent" systems that serve 
users throughout all areas of their daily life. They range from classical technical systems such as household devices, cars, or consumer electronics through mobile apps and services, to advanced service robots in various fields of application. While many of the rather conventional systems already provide multiple modalities to interact with, the most advanced are even equipped with abilities such as perception, cognition, reasoning, and, more recently, social and emotional intelligence. However, the use of such complex technical systems and in particular the actual exploitation of their rich functionality remain challenging and quite often lead to users' cognitive overload and frustration. Companion technologies aim at bridging the gap between the extensive functionality of technical systems and human users' individual requirements, needs, and preferences. They enable the construction of really smart adaptive, flexible, and cooperative technical systems by employing a combination of $\mathrm{AI}$ and $\mathrm{HCI}$ techniques and relying on psychological and neurobiological findings. The special issue "Companion Technologies" of the KI Journal aims at presenting ongoing research, application perspectives, and other insights into an exciting research area emerging across the fields of Artificial Intelligence, Human-Computer Interaction, Cognitive Psychology, and Cognitive Sciences.

Topics of interest include, but are not limited to:

- Computational Models of Cognitive Processes

- Reasoning for Adaptive Systems

- User-Centered Planning

- Multi-modal Emotion and Motivation Recognition

- Knowledge-based Human-Computer Interaction

- Knowledge-based Dialogue Management

- Cooperative and Adaptive Systems.

The KI Journal, published and indexed by Springer, supports a variety of formats including technical articles, project descriptions, surveys, dissertation abstracts, conference reports, and book reviews. Interested authors are asked to contact the guest editors at their earliest convenience:

\section{Prof. Dr. Susanne Biundo-Stephan}

Institute of Artificial Intelligence

Ulm University

89069 Ulm

susanne.biundo@uni-ulm.de

\section{Daniel Höller}

Institute of Artificial Intelligence

Ulm University

89069 Ulm

daniel.hoeller@uni-ulm.de

\section{Pascal Bercher}

Institute of Artificial Intelligence

Ulm University

89069 Ulm

pascal.bercher@uni-ulm.de

\subsection{Semantic Web}

With more and more data available on the web, the use of semantic technologies is the key to making this knowledge accessible to machines. Thirteen years after the last special issue on the Semantic Web, it is time to review again the advances and state-of-the-art in this area. Several new standards for specifying data and schema information in a machine-processable way have emerged: the basic language to describe data, the resource description framework (RDF) with its schema extension RDFS has been revised in 2014; SPARQL became a widely used query language (SPARQL 1.1 standardised in 2013) and now also supports updates, retrieval of entailed query answers, and federation; the Web Ontology Language OWL has been revised and extended to OWL 2; and the rule interchange format RIF (standardized in 2013) allows to express rules in a common format. The recent Linked Open Data movement is based on these standards and makes a vast amount of interlinked resources available in the web and for use in semantically enriched applications. What was still a far-away future when the first Semantic Web special issue appeared became reality: the big search engines now use semantic markup (via RDFa, microdata, or microformats) to improve their search results. With their joint schema.org initiative, Google, Microsoft, and Yahoo! provide a unified schema for the structured description of web page content.

Topics of interest include, but are not limited to:

- Management of semantics and data on the Web, including Linked Data

- Languages, tools, and methodologies for representing and managing semantics and data on the Web

- Database, Information Retrieval, Information Extraction, Natural Language Processing and Artificial Intelligence techniques for the Semantic Web

- Searching and querying the Semantic Web

- Knowledge representation and reasoning on the Web

- Cleaning, quality assurance, and provenance of Semantic Web data, services, and processes

- Semantic Web data analysis

- Ontology-based data access and integration/exchange on the Web

- User Interfaces and interaction with semantics and data on the Web 
- Information visualization of Semantic Web data

- Ontology engineering and ontology patterns for the Web

- Ontology modularity, mapping, merging, and alignment for the Web

- Trust, privacy, and security on the Semantic Web.

The KI Journal, published and indexed by Springer, supports a variety of formats including technical articles, project descriptions, surveys, dissertation abstracts, conference reports, and book reviews.

Interested authors are asked to contact the guest editors at their earliest convenience:
Juniorprof. Dr. Birte Glimm

Institute of Artificial Intelligence

University of Ulm

89069 Ulm

birte.glimm@uni-ulm.de

Prof. Dr. Heiner Stuckenschmidt

Data- and Web Science Research Group

University of Mannheim

68159 Mannheim

heiner@informatik.uni-mannheim.de 